\title{
Conformation of Microcontact-Printed Proteins by Atomic Force Microscopy Molecular Sizing
}

\author{
Adriana Biasco, ${ }^{*}, \dagger$ Dario Pisignano, Blandine Krebs, ${ }^{\dagger}$ Pier Paolo Pompa ${ }^{\dagger}$ \\ Luana Persano ${ }^{\dagger}$ Roberto Cingolani, and Ross Rinaldi \\ National Nanotechnology Laboratory of Istituto Nazionale di Fisica della Materia, \\ c/o Dipartimento di Ingegneria dell'Innovazione, Università di Lecce, via Arnesano, \\ I-73100 Lecce, Italy
}

Received January 3, 2005. In Final Form: March 8, 2005

\begin{abstract}
We investigated the structural changes occurring in proteins patterned via microcontact printing. This was done by molecular sizing using atomic force microscopy to observe the structure of printed individual metalloprotein molecules in the unlabeled and untreated states. We observed that the size of the printed proteins were more than 2 -fold smaller than the native shape, which indicates that some deformations take place upon the contact-assisted adsorption on silanized silicon dioxide. This can be attributed to simultaneously occurring effects, and particularly to the sandwiching between surfaces of very different hydrophilic/hydrophobic properties during contact lithography.
\end{abstract}

\section{Introduction}

Within the past several years, the capability of producing patterns of biological molecules with micrometer-level resolution has attracted increasing interest with respect to their possible use in a wide number of applications. These applications include microarray technologies for genomics and proteomics, medical diagnostics, biological assays and sensors, ${ }^{1}$ molecular electronics, ${ }^{2,3}$ control of cellular adhesion, growth and functionality, ${ }^{4}$ and bacterial detection. ${ }^{5}$ To fabricate biomicropatterns, we can use photografting, inkjet printing, ${ }^{6}$ printing robots,${ }^{7}$ and soft lithography. ${ }^{8}$ However, soft lithography is particularly suitable due to its experimental simplicity, low cost, and extreme flexibility in terms of the employable substrates, solvents, and deliverable molecules. Imprinting processes, ${ }^{9}$ microfluidic networks, ${ }^{10}$ and microcontact printing $(\mu \mathrm{CP})$ succeeded in achieving chemical contrast among different biomolecular monolayers. In particular, $\mu \mathrm{CP}$, which was originally proposed to fabricate self-assembled monolayers (SAMs) of alkanethiolates onto gold, ${ }^{11}$ was soon applied to the synthesis of patterned proteins on surfaces. ${ }^{12}$

Proteins are biological macromolecules comprising one or more chains of amino acids, linked by peptide bonds and folded into a specific three-dimensional configuration (tertiary structure). A protein is biologically functional if

\footnotetext{
* Corresponding author. E-mail: adriana.biasco@unile.it.

$\dagger$ Also at ISUFI, Innovative Materials and Technology School, Università di Lecce, Via Arnesano, I-73100 Lecce, Italy.

(1) Gross, G. W.; Rhoades, B. K.; Azzazy, H. M.; Wu, M. C. Biosens. Bioelectron. 1995, 10, 553. Kane, R. S.; Takayama, S.; Ostuni, E.; Ingber, D. E.; Whitesides, G. M. Biomaterials 1999, 20, 2363.

(2) Martin, A. S.; Sambles, J. R. Phys. Rev. Lett. 1993, 70, 218. Joachim, C.; Gimzewski, J.; Aviram, A. Nature 2000, 408, 541.

(3) Rinaldi, R.; Biasco, A.; Maruccio, G.; Cingolani, R.; Alliata, D.; Andolfi, L.; Facci, P.; De Rienzo, F.; De Felice, R.; Molinari, E. Adv. Mater. 2002, 14, 1453.

(4) Chen, C. S.; Mrksich, M.; Huang, S.; Whitesides, G. M.; Ingber, D. E. Science 1997, 276, 1425. Ito, Y. Biomaterials 1999, 20, 2333.

(5) Howell, S. W.; Inerowicz, H. D.; Regnier, F. E.; Reifenberger, R. Langmuir 2003, 19, 436.

(6) Calvert, P. Chem. Mater. 2001, 13, 3299.

(7) MacBeath, G.; Schreiber, S. L. Science 2000, 289, 1760.

(8) Xia, Y.; Whitesides, G. M. Angew. Chem., Int. Ed. 1998, 37, 550.

(9) Fang, A.; Ng, H. T.; Su, X.; Li, S. F. Y. Langmuir 2000, 16, 5221.

(10) Delamarche, E.; Bernard, A.; Schmid, H.; Michel, B.; Biebuyck, H. Science 1997, 276, 779. Delamarche, E.; Bernard, A.; Schmid, H.;
} Bietsch, A.; Michel, B.; Biebuyck, H. J. Am. Chem. Soc. 1998, 120, 500. it is able to fold into its native state rapidly and reliably. Although much work has been carried out to achieve highthroughput direct printing of biomolecules onto surfaces, the evaluation of the possible structural changes induced by $\mu \mathrm{CP}$ on proteins has not yet been addressed. In particular, the drying process and interaction with the different surfaces involved in the lithography process may have a negative impact on the protein stability, sometimes leading to reversible or irreversible aggregation and loss of functionality.

Although many approaches allow one to investigate the morphology of proteins on solid substrates, ${ }^{13}$ the number of techniques available to quantitatively measure structural changes of proteins at interfaces is limited. Methods such as total internal reflectance fluorescence provide a measure of the average properties of all the adsorbed proteins but give no information about the individual molecules. Other techniques such as X-ray photoelectron spectroscopy require a vacuum environment as well as sample preparation procedures that alter the physiological conditions of the protein. However, scanning force microscopy allows one to investigate the protein adsorption on a surface by imaging single biomacromolecules ${ }^{14-16}$ with precise molecular sizing.

In this paper, we report the investigation of the conformational morphology of microcontact-printed proteins by atomic force microscopy (AFM) molecular sizing. We observed that the size of individual printed metalloprotein molecules in the unlabeled and untreated state

(11) Kumar, A.; Whitesides, G. M. Appl. Phys. Lett. 1993, 63, 2002.

(12) Bernard, A.; Delamarche, E.; Schmid, H.; Michel, B.; Bosshard, H. R.; Biebuyck, H. Langmuir 1998, 14, 2225. James, C. D.; Davis, R. C.; Kam, L.; Craighead, H. G.; Isaacson, M.; Turner, J. N.; Shain, W. Langmuir 1998, 14, 741. Bernard, A.; Renault, J. P.; Michel, B.; Bosshard, H. R.; Delamarche, E. Adv. Mater. 2000, 12, 1067. Sgarbi, N.; Pisignano, D.; Di Benedetto, F.; Gigli, G.; Cingolani, R.; Rinaldi, R. Biomaterials 2004, 25, 1349.

(13) Bergkvist, M.; Carlsson, J.; Oscarsson, S. J. Phys. Chem. B 2001 105, 2062 and references therein.

(14) Lin, J. N.; Drake, B.; Lea, A. S.; Hansma, P. K.; Andrade, J. D. Langmuir 1990, 6, 509.

(15) Binning, G.; Quate, C. F.; Gerber, C. Phys. Rev. Lett. 1986, 56 930.

(16) Cavalleri, O.; Natale, C.; Stroppolo, M. E.; Relini, A.; Cosulich, E.; Thea, S.; Novi, M.; Ghiozzi, A. Phys. Chem. Chem. Phys. 2000, 2, 4630 . 


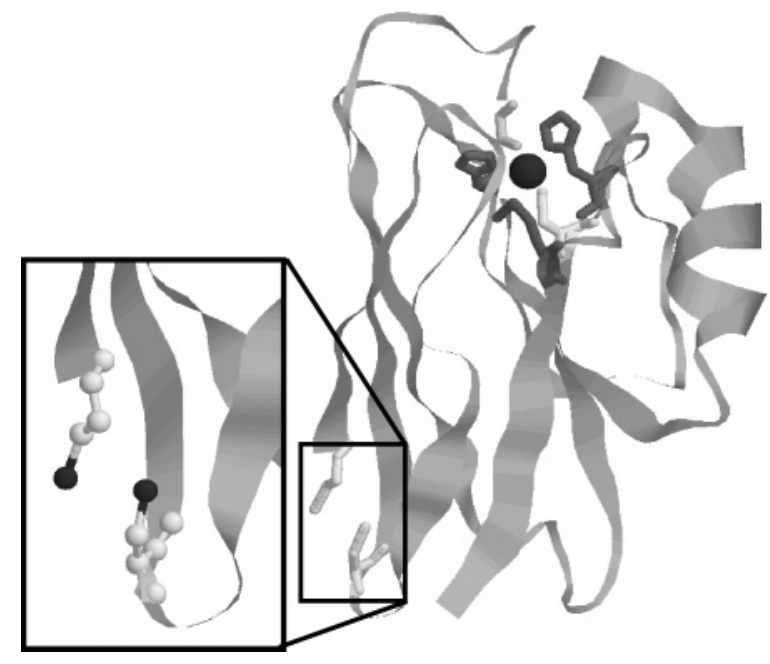

Figure 1. Molecular structure of the Az metalloprotein. Inset: disulfide bridge of the molecule. The dark balls in the inset stand for the sulfur atoms involved in the bond.

was more than 2 -fold smaller than the native shape. This indicates that some deformations take place upon the contact-assisted adsorption on silanized $\mathrm{SiO}_{2}$. As a prototype system, we used printed SAMs of the blue copper protein Pseudomonas aeruginosa Azurin (Az) (Figure 1). This is one of the best characterized redox metalloproteins, ${ }^{17}$ which mediates electron transfer in denitrifying bacteria by transporting electrons from cytochrome $c_{551}$ to nitrite reductase in the electron transport chain of respiratory phosphorylation. ${ }^{18} \mathrm{Az}$ is used to fabricate biomolecular electronic devices, ${ }^{3}$ and its adsorption onto both gold and functionalized oxide surfaces has been widely investigated. ${ }^{16,19}$ The average protein size measured by X-ray crystallography is about $3.5 \times 3.5 \times 4.4 \mathrm{~nm}^{3}{ }^{20}$

\section{Experiment}

$\mathrm{Az}$ and the anti-Az serum (primary antibody) were supplied by the Metalloprotein group, Gorlaeus Laboratory, in Leiden, The Netherlands. The protein was diluted in $20 \mathrm{mM}$ Hepes buffer, $\mathrm{pH} 4.6$, to final concentrations of $10^{-8}$ and $10^{-5} \mathrm{M}$. A surface organic modifier (3-mercaptopropyltrimethoxysilane, 3-MPTS) was used to chemically bind the protein. A uniform hydrophilic monolayer with root-mean-square $(\mathrm{rms})$ roughness of $0.3 \mathrm{~nm}^{21}$ was produced by substrate incubation in a 3-MPTS solution. $\mathrm{SiO}_{2}$ modification is required because unmodified $\mathrm{SiO}_{2}$ acquires some charges when exposed to ionic solutions, which can lead to protein adsorption kinetics dominated by electrostatics. This would prevent control over the specific molecule orientation, which is possible only by chemical adsorption. On the other hand, when modified $\mathrm{SiO}_{2}$ is used, the Az molecules adsorb with high binding specificity via the disulfide bond, resulting in a chemically driven molecular orientation. ${ }^{16}$

Freshly cleaved $1 \mathrm{~cm}^{2}$ pieces of $\mathrm{SiO}_{2}$ were washed with an acetone and propanol cascade, and then incubated overnight in acidic solution $\left(\mathrm{H}_{2} \mathrm{O}_{2}: \mathrm{H}_{2} \mathrm{SO}_{4}=1: 3\right)$ to remove organic contaminants. The substrates were sonicated three times for $5 \mathrm{~min}$ in water at $25{ }^{\circ} \mathrm{C}$, and then dried with a blast of $\mathrm{N}_{2}$ gas to remove the bulk water, thus preventing undesirable polymerization of the 3-MPTS. All the surfaces were used immediately after cleaning. The covalent bonding of the silane groups was achieved

(17) Adman, E. T.; Stenkamp, R. E.; Sieker, L. C.; Jensen, L. H. J. Mol. Biol. 1978, 123, 35.

(18) Webb, M. A.; Kwong, C. M.; Loppnow, G. R. J. Phys. Chem. B 1997, $101(25), 5062$.

(19) Schnyder, B.; Kotz, R.; Alliata, D.; Facci, P. Surf. Interface Anal. 2002, 34, 40.

(20) Nar, H.; Messerschmidt, A.; Huber, R.; van de Kamp, M.; Canters, G. W. J. Mol. Biol. 1991, 221, 765 .

(21) Biasco, A.; Maruccio, G.; Visconti, P.; Bramanti, A.; Calogiuri, P.; Cingolani, R.; Rinaldi, R. Mater. Sci. Eng. C 2004, 24, 563.

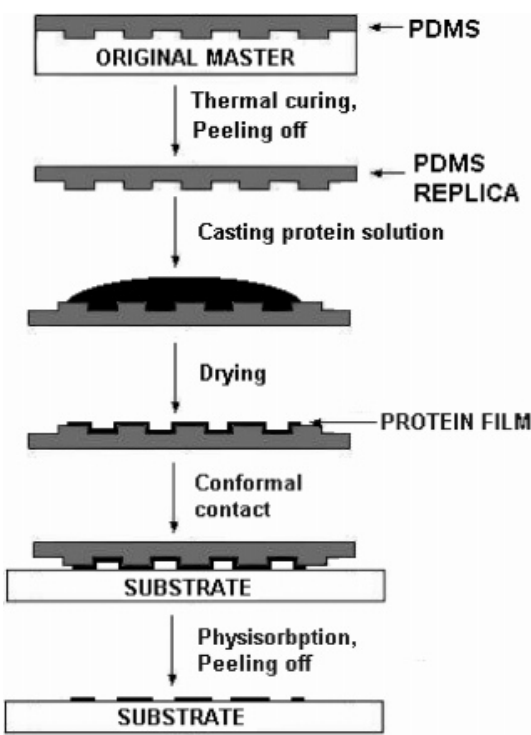

Figure 2. Schematic diagram of performed $\mu \mathrm{CP}$ process (features not to scale).

by incubation ( $15 \mathrm{~min}$ ) of the substrates in the 3-MPTS solution. This procedure was followed by a 1 min sonication in ethanol, to remove unbound silane compounds, and drying with a $\mathrm{N}_{2}$ flux. The substrates were then stored under vacuum prior to initiating contact with the protein. Elastomeric replicas of hard masters are produced by in situ polymerization of poly(dimethylsiloxane) (PDMS; Sylgard 184, A:B 1:9, Dow Corning). The polymerization was done at $140{ }^{\circ} \mathrm{C}$ for $15 \mathrm{~min}$. For the experiments with hydrophilic PDMS, we exposed the patterned side of the elastomer to $\mathrm{O}_{2}$ plasma treatment. A few drops of the protein solutions $\left(10^{-8-} 10^{-5} \mathrm{M}\right)$ were cast on the patterned side of the replica and gently dried with an $\mathrm{N}_{2}$ stream. The protein-coated elastomeric element was placed onto the prepared substrates under its own weight, immediately after the solvent evaporation. After peeling off of the replicas, the patterned substrates were vigorously rinsed several times to remove the unattached protein molecules.

Immunofluorescence micrographs of the printed patterns were collected by a Leica fluorescence inverted microscope fitted with a monochrome camera, AxioCam MR (Zeiss). The AFM experiments were performed with a BioScope microscope (Digital Instruments) equipped with a $90 \mu \mathrm{m}$ scanner and operating by tapping mode in air. We used $\mathrm{Si}_{3} \mathrm{~N}_{4}$ tips with a $10 \mathrm{~nm}$ nominal curvature radius and a scanning rate of $1 \mathrm{~Hz}$. The intrinsic fluorescence of $\mathrm{Az}$ in solution and of incubated films was determined by a Perkin-Elmer LS 50B spectrofluorimeter.

\section{Results and Discussion}

Figure $3 \mathrm{~b}$ is a fluorescent photograph showing the resulting $\mu \mathrm{CP}$ pattern of $\mathrm{Az}$ after the polyclonal antibody incubation. It clearly indicates that the proteins were deposited in a striped geometry, which is identical to the master pattern (Figure 3a). Approximately $9 \mu \mathrm{m}$ sets of uniform, wide parallel tracks were observed. They present sharp edge interfaces, which indicates a nonsignificant protein diffusion on the substrate after the removal of the mold. Therefore, the resolution of the printed patterns can be certainly reduced with respect to the result reported here, depending on the starting master.

In fact, immunofluorescence microscopy gives information about the fidelity of the transfer of the pattern, but it does not allow a full investigation of the protein orientation or of its retention of functionality, although some quantitative fluorescence studies of printed antibodies were recently reported. ${ }^{22}$ In particular, the use of polyclonal primary antibodies, similarly to most of the to

(22) Graber, D. J.; Zieziulewicz, T. J.; Lawrence, D. A.; Shain, W.; Turner, J. N. Langmuir 2003, 19, 5431. 

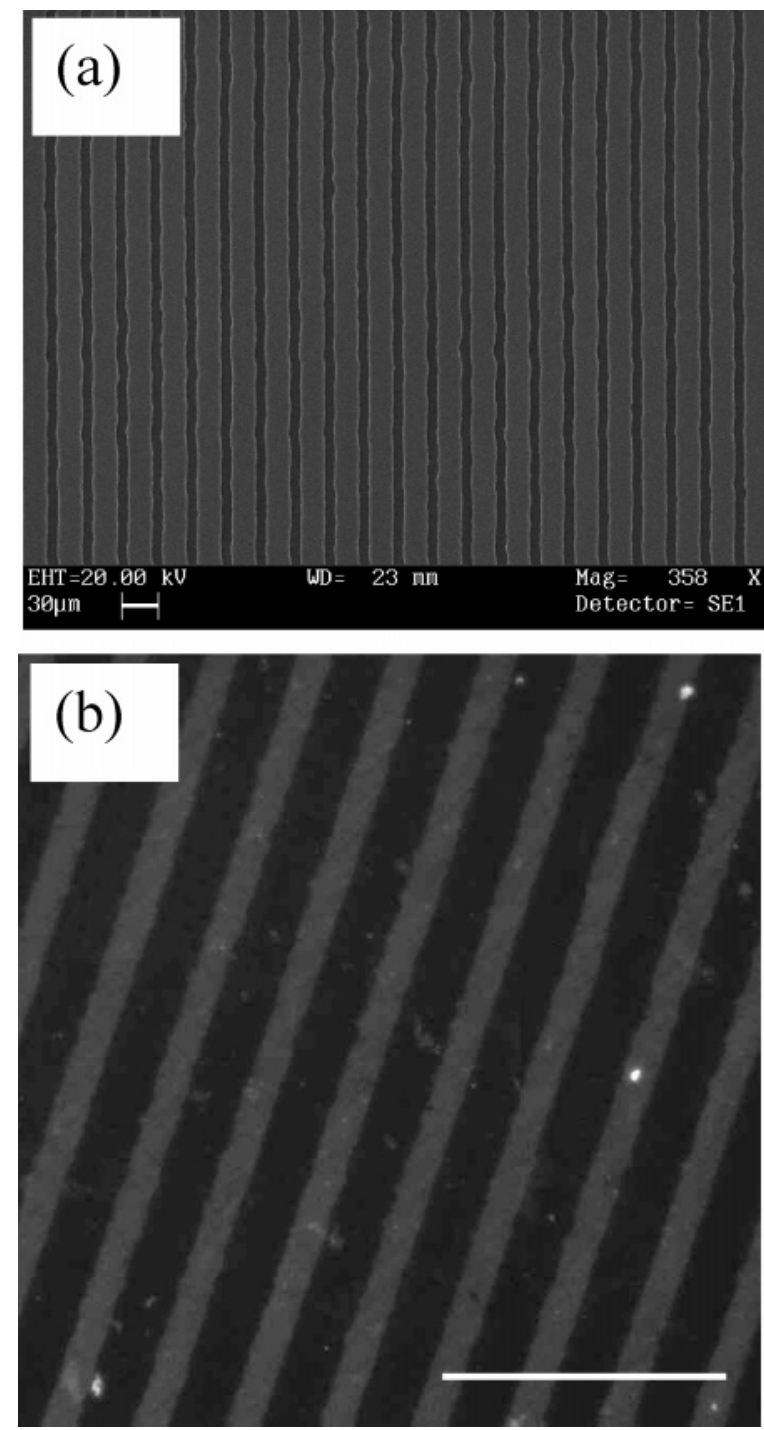

Figure 3. (a) Master pattern used for $\mu \mathrm{CP}$ experiments. (b) Immunofluorescence micrograph of the obtained $\mathrm{Az}$ pattern. The white bar indicates $100 \mu \mathrm{m}$ length. The printed stripes are about $9 \mu \mathrm{m}$ wide, faithfully reproducing the master geometry.

date reported $\mu \mathrm{CP}$ experiments, allows one to recognize a variety of epitopes on the antigen, thus being quite tolerant of changes of the proteins in terms of conformation, orientation, or slight denaturation.

To investigate the conformation of proteins in the patterned regions, tapping-mode AFM images were collected in air on unlabeled samples. At large scan size (Figure 4) they confirm the homogeneity and the welldefined edges of the $\mu \mathrm{CP}$ pattern. At small scan size (inset of Figure 4), it is possible to image single proteins and to characterize their size distribution within the printed monolayer. In the case of globular proteins, such as Az, AFM provides poor information about the protein orientation because the molecular axes are indistinguishable from each other. However, it can discriminate whether the proteins undergo deformations or breaks as a consequence of the lithography process. In fact, the tipsample convolution effect resulting from the physical dimension of the tip creates a low lateral resolution on small globular proteins. On the contrary, height measurements are almost unaffected by the convolution effect; therefore, high resolution can be reached in this dimension. Indeed, the height measured by AFM can be slightly smaller than the actual molecular size, due to the finite

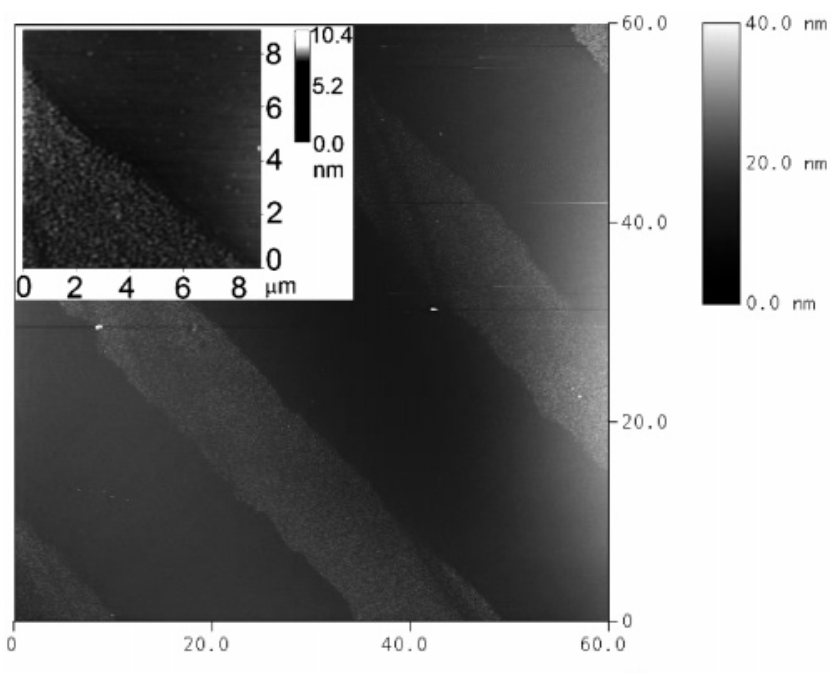

$\mu \mathrm{m}$

Figure 4. Topography image of the printed Az layer. The inset is a magnification of the bottom-left area.

distance between two consecutive scanning lines. The maximum underestimate $(\Delta h)$ of the molecule height is ${ }^{23}$

$$
\Delta h=R+r-\sqrt{(R+r)^{2}-\Delta x^{2}}
$$

where $R$ indicates the radius of the globular protein, $r$ indicates the radius of the tip (nominally $10 \mathrm{~nm}$ ), and $\Delta x$ stands for the maximum horizontal displacement between the top of the protein and the nearest probe position. By considering a $2 \times 2 \mu \mathrm{m}^{2}$ image with $512 \times 512$ sampling points, one obtains a height discrepancy of about $1.6 \AA$ for a 4-nm-diameter globular protein. This discrepancy is about $4 \%$ of the molecule size.

The measured rms roughness of $\mathrm{Az}$ printed onto silanized $\mathrm{SiO}_{2}$ does not exceed $1 \mathrm{~nm}$, thus being significantly smaller than the value $(3 \mathrm{~nm})$ found for $\mathrm{Az}$ monolayers obtained by protein adsorption from cast bulk solution. ${ }^{21}$ In order to have deeper insight into this evidence, and to understand how the protein tertiary structure is affected by the lithography, we analyzed the distribution of measured heights from a significant number of samples. The sizes of individual proteins were measured by subtracting the height of the local background (i.e., the unpatterned substrate) from the maximum height of the molecules. The fit of the experimental data was performed repeatedly by means of a combination of Gaussian functions, ${ }^{24}$ where the centers of the Gaussians were taken as the measured molecular dimensions. The analysis, carried out on several independent AFM images, yielded a distribution of measured heights resulting from the sum of three different contributions (Figure 5). The best-fit parameters of some Gaussian distributions of $\mathrm{Az}$ population are reported in Table 1.

Parts a and b of Figure 5 show the results from two different printed Az/3-MPTS/SiO ${ }_{2}$ samples: The AFM molecular sizing reveals that the main protein population has a height around 1.45 and $1.97 \mathrm{~nm}$, respectively, which is significantly smaller than the native size. This clearly provides evidence that some deformations of the protein occur as a consequence of the interaction of the biomolecule with the substrate, or of the $\mu \mathrm{CP}$ process, or of both.

(23) Waner, M. J.; Gilchrist, M.; Schindler, M.; Dantus, M. J. Phys. Chem. B 1998, 102, 1649 .

(24) Brusatori, M. A.; Van Tassel, P. R. J. Colloid Interface Sci. 1999 219,333 
Table 1. Shape Parameters of the Fitting Gaussian Functions Shown in Figure 5

\begin{tabular}{ccccc}
\hline substrate & species & height [nm] & fwhm [nm] & probability [\%] \\
\hline modified $\mathrm{SiO}_{2}$ (Figure 5a) & 1 & $0.80 \pm 0.08$ & $0.62 \pm 0.06$ & 14 \\
& 2 & $1.45 \pm 0.02$ & $0.70 \pm 0.06$ & 64 \\
modified $\mathrm{SiO}_{2}$ (Figure 5b) & 3 & $2.15 \pm 0.11$ & $0.80 \pm 0.08$ & 22 \\
& 1 & $1.30 \pm 0.10$ & $0.80 \pm 0.05$ & 27 \\
$\mathrm{Au}$ (Figure 5c) & 2 & $1.97 \pm 0.09$ & $0.90 \pm 0.14$ & 44 \\
& 3 & $2.94 \pm 0.11$ & $1.20 \pm 0.09$ & 29 \\
& 1 & $2.38 \pm 0.16$ & $1.52 \pm 0.05$ & 54 \\
& 3 & $4.05 \pm 0.15$ & $1.55 \pm 0.07$ & 23
\end{tabular}
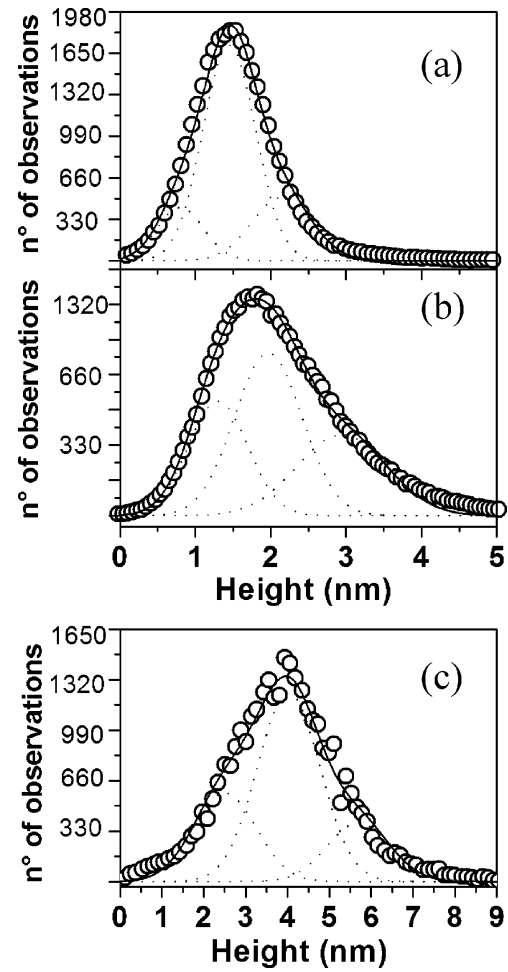

Figure 5. Population of Az printed molecules vs protein height measured by AFM, and corresponding fit (solid line) by the sum of three Gaussian contributions (dotted lines), for two different 3-MPTS/ $\mathrm{SiO}_{2}$ samples ( $\mathrm{a}$ and $\mathrm{b}$ ) and for a gold substrate (c). In (c), the additional peaks in the height distribution indicate the existence of two different protein species (mainly deriving from unfolded structures), possibly suggesting the formation of aspecific aggregates (average size $5.6 \mathrm{~nm}$ ) and flattened proteins (average size $2.4 \mathrm{~nm}$ ).

Proteins in solutions are subjected to intramolecular forces stabilizing their helical conformations. Upon coming into contact with a solid substrate, the interactions (chemical or electrostatic) between the substrate surface and the peptide side chains provide the primary driving force for adsorption. ${ }^{25}$ The balance between these binding interactions and the intramolecular stabilizing forces determines the extent to which the protein loses its own helicity upon adsorption, with a possible consequent alteration of the tertiary structure. ${ }^{24}$ These conformational changes range from slight shape modifications to complete denaturation, occurring on time scales between a few minutes and some hours. ${ }^{26,27}$ In particular, it has been suggested that the adsorption onto hydrophobic surfaces can result in heavy structural modifications due to the protein tendency to expose inner hydrophobic groups. ${ }^{26}$

(25) Marsh, R. J.; Jones, R. A. L.; Sferrazza, M. Colloids Surf., B: Biointerfaces 2002, 23, 31.

(26) Moulin, A. M.; O’Shea, S. J.; Badley, R. A.; Doyle, P.; Welland, M. E. Langmuir 1999, 15, 8776.

(27) Holt, S. A.; McGillivray, D. J.; Poon, S.; White, J. W. J. Phys. Chem. B 2000, 104, 7431.

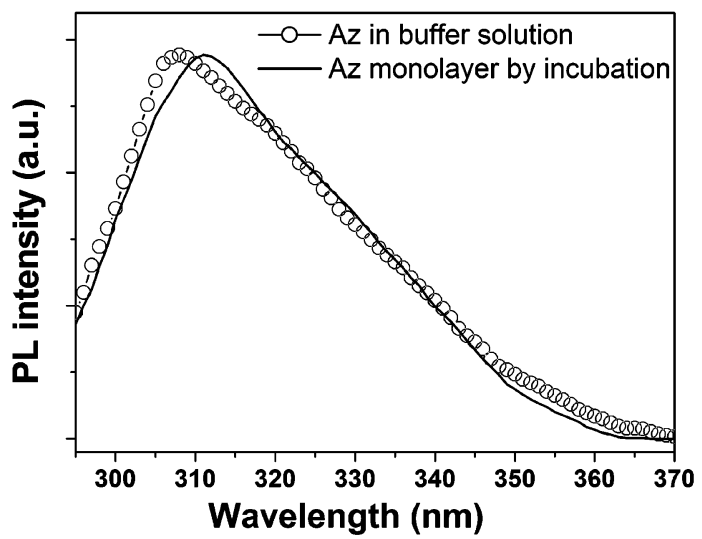

Figure 6. Normalized intrinsic fluorescence of $\mathrm{Az}$ in buffer solution (open circles) and in monolayers chemisorbed by incubation (continuous line).

On the other hand, soluble proteins adsorbed onto hydrophilic substrates are more likely to retain their native conformation. ${ }^{25}$ In addition, "compact" globules, such as Az, are more likely to maintain their native structure during adsorption than deformable flexible proteins. The high rigidity of the adsorbed molecules induced by the disulfide bond with the surface is expected to further disfavor large conformational changes. This is confirmed by a number of previous experiments of adsorption on incubated surfaces, which were investigated by atomic force microscopy and scanning tunneling microscopy (STM). ${ }^{28}$ These studies indicated that there were no protein conformation changes.

In particular, the morphological, electronic, and spectroscopic investigation of Az monolayers, immobilized on functionalized $\mathrm{SiO}_{2}$ (by casting or incubation from buffer solutions), confirm that the proteins retain their correct conformational and functional states. AFM measurements performed in ambient conditions (in air) on individual adsorbed Az molecules show that the great majority of the protein population is not affected by denaturation processes directly induced by the interaction with the substrate surface. We arrive at this conclusion because the main histogram band is centered around $\sim 4 \mathrm{~nm} .{ }^{29}$ After analyzing the intrinsic fluorescence spectrum of the chemisorbed $\mathrm{Az}$ monolayer, we obtained further evidence that the emission spectrum of the immobilized protein is similar to that of the free protein in buffer (Figure 6). The tryptophan residue (Trp48), responsible for the protein fluorescence, is embedded in similar locations both in the native protein and in the immobilized protein. This finding indicates that the microenvironment surrounding the residue is correctly retained after adsorption on the substrate surface by incubation. Such an effect is likely

(28) Facci, P.; Alliata, D.; Cannistraro, S. Ultramicroscopy 2001, 89, 291. Davis, J. J.; Hill, H. A. O. Chem. Commun. 2002, 393.

(29) Pompa, P. P.; Biasco, A.; Frascerra, V.; Calabi, F.; Cingolani, R.; Rinaldi, R.; Verbeet, M. Ph.; De Waal, E.; Canters, G. W. J. Chem. Phys. 2004, 121, 10325. 
related to the capability of the molecule to preserve its hydration shell even under ambient conditions. This mechanism is also confirmed by STM experiments carried out in liquid and in air on gold-chemisorbed Az. ${ }^{29}$ Indeed, the retention of the hydration shell protects the superficial charge distribution during the dehydration process, thus preserving the electron transfer function in nonphysiologically adsorbed proteins. As a result, the air ambient interacting does not deteriorate the structure and function of $\mathrm{Az}$, even though it often affects the active folding of water-soluble proteins. Finally, well-retained intrinsic fluorescence of proteins adsorbed by incubation (Figure 6) also allows one to exclude the possibility of significant damages induced by the amount of salt and buffer that might remain on the surface after washing. For these reasons, we conclude that direct denaturation as a consequence of interaction with the functionalized $\mathrm{SiO}_{2}$ surface (also with air exposure) is not possible in the case of Az. Not withstanding this, we do observe a significant deformation in the $\mathrm{Az}$ patterned by $\mu \mathrm{CP}$, which can be due to the effect of the sandwich-like contact between the coated elastomer and the target substrate, and also to the complex electrostatic, steric, and hydrophilic/hydrophobic protein-surface interactions.

A flattening effect of the pressure applied on the $\mathrm{Az}$ molecules during the lithography process can be ruled out. During the $\mu \mathrm{CP}$, the protein-coated elastomeric element (density of about $0.95 \mathrm{~g} / \mathrm{cm}^{3}$ ) is placed on the substrate under its own weight and very small applied pressures $\left(\sim 1 \mathrm{~g} / \mathrm{mm}^{2}{ }^{30}\right)$. The force on the proteins, by a 1-mm-thick replica with an overall area of $1 \mathrm{~cm}^{2}$ and a $50 \%$ filling-density pattern, is therefore less than $10^{-6}$ $\mathrm{nN}$. This force is many orders of magnitude smaller than the load $(1-10 \mathrm{nN})$ needed to mechanically compress the Az molecules, determined by recent conductive-probe AFM experiments. ${ }^{31}$

Therefore, the possible flattening mechanisms have to be looked for among (i) the drying step on the elastomeric

(30) Csus, G.; Kunzler, T.; Feldman, K.; Robin, F.; Spencer, N. D. Langmuir 2003, 19, 6104.

(31) Zhao, J.; Davis, J. J. Nanotechnology 2003, 14, 1023. surface, ${ }^{32}$ which can alter the protein structure more heavily than casting from solution, ${ }^{3}$ and (ii) the sandwiching between surfaces of very different hydrophilic/ hydrophobic properties. ${ }^{33}$ Interestingly, the height distribution of Az molecules printed onto gold, $\mathrm{Au}(111)$, show instead a main molecular species, accounting for approximately $54 \%$ of the protein population, centered around $4 \mathrm{~nm}$ (Figure 5c). This could suggest a detrimental effect of the organic modifiers on the protein structure, which is instead well-preserved on bare gold substrates. Such a flattening effect could be irreversibly enhanced by the $\mu \mathrm{CP}$ interval, during which the protein can be strongly attracted by the hydrophilic - $\mathrm{SH}$ groups. In particular, sandwiching the protein between surfaces of very different hydrophilic/hydrophobic properties during the lithographic contact can strongly affect the protein conformation.

In conclusion, our findings show a reduction of the metalloprotein native size as a consequence of the $\mu \mathrm{CP}$. This can be attributed to the energy constraints due to the different surfaces involved in contact lithography. These constraints are represented by strong interactions between the hydrophilic surface amino acids and the $\mathrm{SiO}_{2}$ modified by the $-\mathrm{SH}$ terminations of the employed silane layer. We believe that the scanning force microscopy molecular sizing, here employed for printed protein films, can enable an in-depth investigation of the structural changes of biomolecules patterned by soft lithography.

Acknowledgment. Support for this research was provided by the Italian Istituto Nazionale di Fisica della Materia and Minister of University and Scientific Research (FIRB-Nanodispositivi Molecolari Prot. RBNEO1YSR8), and by the EU (SAMBA Project). We thank Dewan Andrews for useful suggestions.

LA050010J

(32) Tan, J. L.; Tien, J.; Chen, C. S. Langmuir 2002, 18, 519.

(33) We also tried to use plasma-treated hydrophilic replicas for $\mu \mathrm{CP}$, but this did not lead to a good pattern transfer on the target substrate, likely because of the competing attraction of the hydrophilic elastomer surface toward the Az molecules (see ref 32 ). 